\title{
La evaluación de competencias en una experiencia de formación dual de maestros: diferencias y semejanzas entre tutores de escuela y de universidad
}

\author{
The competences assessment in a dual training experience of teachers: differences and \\ similarities between school and university tutors
}

\section{A avaliação de competências em uma experiência de formação dual de professores: diferenças e semelhanças entre os tutores da escola e os da universidade}

\author{
Jordi Coiduras Rodríguez, ${ }^{a}$ Georgina París Mañas, ${ }^{a b}$ Cristina Torrelles Nadal, ${ }^{a c}$ \\ Xavier Carrera Farran ${ }^{a d}$
}

${ }^{a}$ Universidad de Lleida. Departamento de Pedagogía y Psicología, Cataluña, España

Telf.: 0034973706 579. Correo electrónico: coiduras@ pip.udl.cat

bCorreo electrónico: paris@pip.udl.cat

cCorreo electrónico: ctorrelles@pip.udl.cat

${ }^{\mathrm{d} C}$ Correo electrónico: carrera@pip.udl.cat

\begin{abstract}
RESUMEN
La colaboración necesaria entre universidad y escuela en la formación inicial de maestros ${ }^{1}$ está en el centro de este estudio de caso. Ambos escenarios contribuyen a la construcción de la profesionalidad, la adquisición y desarrollo de las competencias docentes desde misiones institucionales distintas. Con el inicio de una experiencia de formación dual, en un dispositivo pedagógico de integración de aprendizajes, se constatan diferencias entre las evaluaciones de los tutores universitarios y los tutores escolares. Con el interés de explicar estas valoraciones se contrastan en su dimensión global y en relación a la competencia metodológica. Los resultados pueden explicarse principalmente por la diferencia de roles de los tutores: por un lado, desde la socialización profesional en la colaboración en la actividad propiamente escolar (tutores escolares) y, por otro, desde una actividad más propia del análisis, comprensión, relación con los marcos teóricos y reflexión demandados desde la universidad (tutores universitarios).

Palabras clave: formación de docentes, formación dual, tutoría y evaluación.
\end{abstract}

\begin{abstract}
The need for schools and universities to work together in the initial training of teachers lies at the centre of this case study. These two institutions help to build professionalism and to promote the acquisition and development of teaching skills through their respective missions. With the start of a dual training experience, within a pedagogic approach based on the integration of different learning styles, we have noted differences between the ways that university tutors and school tutors make evaluations. To explain these evaluations, we have compared and contrasted them at the global scale and with reference to methodological competences. The results obtained can mainly be explained by the different roles that tutors play in these two contexts. In the case of school tutors, this involves professional socialization in collaboration in school-centred activities. In the other case, the activity of university tutors is more closely related to analysis and understanding, theoretical frameworks and acts of reflection.
\end{abstract}

Key words: teacher training, dual training, tutoring and evaluation.

Para facilitar la lectura se evita la utilización continuada de la duplicidad de género. Así, cada vez que se cita maestro, tutor, estudiante, alumno, etc. se entiende que hace referencia a ambos géneros, sin que ello implique ningún tipo de consideraciones discriminatorias ni valoración peyorativa. 


\section{RESUMO}

A colaboração necessária entre universidade e escola na formação inicial dos professores é a questão principal deste estudo de caso. Ambos cenários contribuem para a construção da profissionalidade, para aquisição e desenvolvimento de competências docentes a partir de diferentes missões institucionais. Com o início de uma experiência de formação dual, em um dispositivo pedagógico de integração de aprendizagens, constatam-se diferenças entre as avaliações de tutores universitários e as dos tutores escolares. Com o interesse de explicar tal valorização, estas são contrastadas em sua dimensão global e em relação à competência metodológica. Resultados podem ser explicados, principalmente, pela diferença de papéis desenvolvidos pelos tutores: por um lado, a partir da socialização profissional da colaboração na atividade escolar (tutores escolares) e, por outro, a partir da atividade mais própria de análise, compreensão, relação com campos teóricos e reflexão exigida desde a universidade (tutores universitários).

Palavras-chave: formação de professores, formação dual, tutoria e avaliação.

\section{LA ESCUELA, ESPACIO DE FORMACIÓN DE MAESTROS}

Distintos autores (Danielson, 2013; Fernández-Enguita, Mena y Rivere, 2010) han relacionado el éxito del sistema educativo de un país con la formación de sus docentes. Desde este análisis la mejora de la formación para la enseñanza debería ser, en las agendas gubernamentales, una estrategia esencial para el progreso en los resultados de aprendizaje en educación primaria y secundaria. También para las universidades en su compromiso de servicio público. En España, los resultados de los informes internacionales PISA² (OCDE, 2012) y nacionales (Martínez y Albaigés, 2013) han puesto en evidencia los insatisfactorios resultados de aprendizaje en las etapas no universitarias. Más allá de la adecuación de estas evaluaciones a los contextos sociológicos, económicos y tradiciones pedagógicas existe un consenso amplio sobre, al menos, la buena estimación de estos índices. Ante esta realidad la universidad debe sentirse interpelada como parte de la solución con un papel principal en la formación de los docentes. Las investigaciones revelan la calidad educativa como el factor más poderoso en el rendimiento infantil, lo que correlaciona positivamente con los resultados del alumnado (American Council on Education, 1999; Comisión Europea, 2007). En esta dirección se ha identificado la formación con experiencias educativas de calidad, como el factor clave para adquirir la competencia inicial necesaria para la inserción profesional.

En el estado español, con la reforma universitaria derivada de la construcción del Espacio Europeo de Educación Superior (EEES), los estudios para la docencia en educación infantil y primaria se han prolongado un año. Esto ha hecho posible una formación más extensa, coherente con el reconocimiento y prestigio que la profesión reclamaba. La ampliación junto a la adopción del enfoque competencial han contribuido en el diseño de planes de estudio con una mayor actividad formativa de los estudiantes en los centros escolares, escenarios imprescindibles para la adquisición de las competencias docentes en la formación inicial. La actividad en los contextos profesionales responde a la necesidad de desarrollar durante la formación inicial la identidad y profesionalidad. El papel de la escuela y sus docentes, con este objetivo, se reconoce fundamental por facilitar la experiencia, acompañar la adquisición de un cierto dominio de conocimientos y habilidades, hacer posible la toma de decisiones y la reflexión sobre la práctica.

Informe PISA: Informe del Programa Internacional para la Evaluación de Estudiantes de la Organización para la Cooperación y el Desarrollo Económicos (OCDE). 
En las últimas décadas la alternancia ha experimentado, paralelamente a su reconocimiento, nuevas adhesiones como modalidad formativa o caracterización de un determinado plan de estudios. El partenariado entre la institución formativa y la empresa se identifica como fuente de desarrollo beneficiosa para el conjunto de los actores (Hoeckel, 2008).

\section{INTEGRACIÓN DE APRENDIZAJES Y FORMACIÓN DUAL}

La relación entre ambas experiencias formativas, universitaria y escolar, requiere despegarse de una visión exclusivamente academicista, como otra forma de tecnicismo, según la cual con la mera adquisición de rutinas, habilidades y solución de problemas sería suficiente parar abordar situaciones educativas complejas (Angulo y Redón, 2011). Esto conlleva la necesaria articulación de ambas, dándose sentido mutuo en su intersección. Las experiencias de educación dual $^{3}$ en las formaciones de carácter profesionalizador responden a una lógica de eficiencia formativa, con una mayor participación de los entornos laborales particulares. Esto es especialmente adecuado en la formación para la educación, cuando el ejercicio de la profesión requiere un dominio práctico y un cierto grado de racionalización del proceso de trabajo.

Uno de los principales retos de la formación en distintos escenarios se sitúa en la integración de aprendizajes. El estudiante suele experimentar tensiones derivadas de las diferencias y exigencias de los dos entornos, así como de la dificultad en vincular los marcos teóricos con las vivencias prácticas. La transformación de estas tensiones en conocimiento práctico requiere una articulación comprensiva entre experiencia y conocimiento, donde el saber disciplinar favorezca la comprensión de diversas realidades y el ejercicio profesional adquiera sentido y se transforme en conocimiento.

Distintos investigadores han analizado el acercamiento entre el mundo profesional y el universitario, para concretar actuaciones de complementariedad y confrontación de los aprendizajes adquiridos en ambos contextos (Armengol et al., 2013). En esta línea, parece necesario contrastar experiencias particulares con otras de carácter teórico y general para estudiar su eficiencia.

\section{TUTORÍA Y EVALUACIÓN DE COMPETENCIAS}

El acompañamiento ha sido definido como un atributo (Maubant, 2009) fundamental de la formación dual para que el aprendiz articule, conecte e integre los aprendizajes derivados de sus experiencias. Tiene una función de mediación experta, como apoyo a la comprensión e interpretación de los componentes clave en la práctica docente. Con el acompañamiento de los tutores escolares los estudiantes se sienten apoyados en una situación de ejercicio profesional compleja, ven incrementada su confianza, mejoran los procesos de reflexión y su capacidad en la resolución de problemas. Portelance et al. (2008) han definido competencias específicas, cuyo ejercicio promovería el aprovechamiento de

Cedefop define formación en dual o en alternancia como aquella que combina periodos lectivos en la institución formativa con periodos de ejercicio en el contexto profesional. La alternancia puede tener lugar a intervalos semanales, mensuales o anuales. En función del país, de la profesión y del reglamento aplicable, los participantes pueden tener una relación contractual con el empleador y percibir una remuneración (Cedefop, 2008). 
Estudios Pedagógicos, vol. XL, Número Especial 1: 29-48, 2014

LA EVALUACIÓN DE COMPETENCIAS EN UNA EXPERIENCIA DE FORMACIÓN DUAL DE MAESTROS:

DIFERENCIAS Y SEMEJANZAS ENTRE TUTORES DE ESCUELA Y DE UNIVERSIDAD

las experiencias y el desarrollo inicial de la profesión. En esta investigación, en QuebecCanadá, los autores obtienen un conjunto de orientaciones y competencias propias de la tutoría a considerar en los programas de formación (Tabla 1).

Tabla 1. Orientaciones y competencias para la formación de los tutores universitarios y escolares (Portelance et al., 2008)

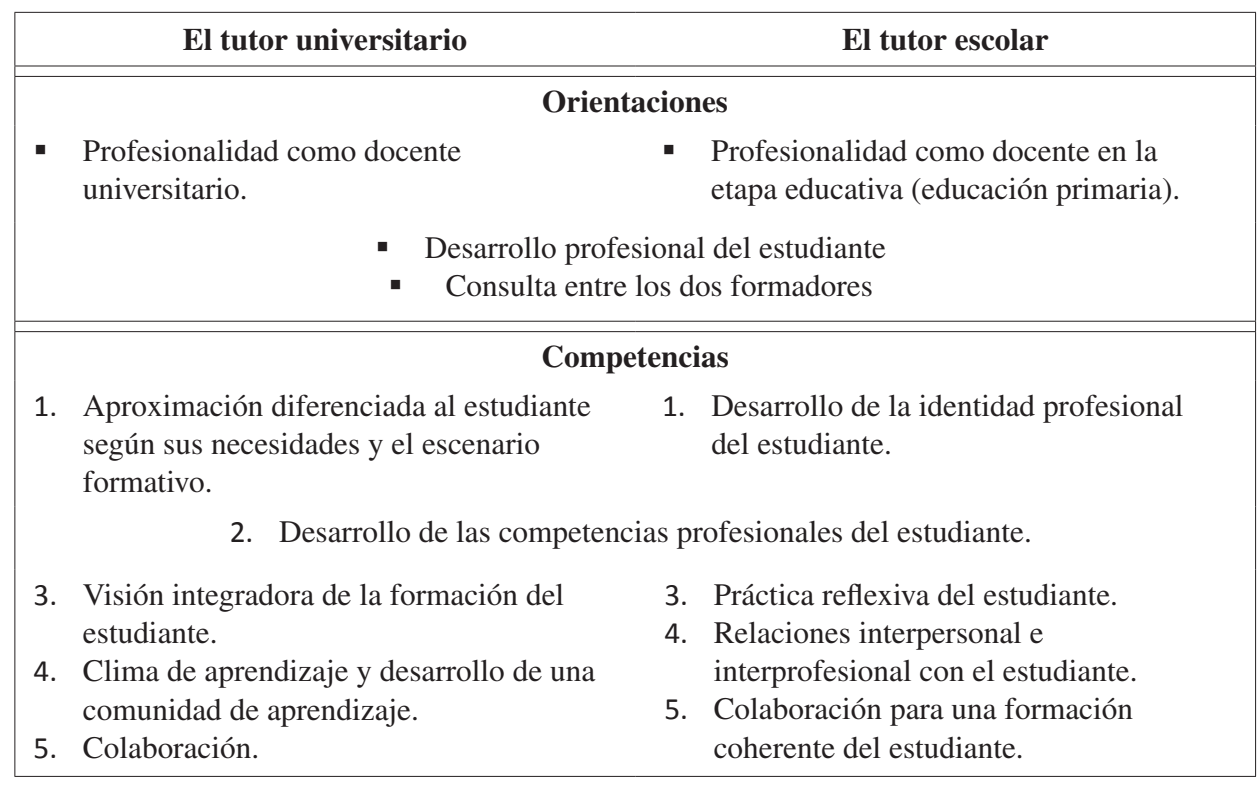

Así como Portelance presenta entre las competencias del tutor escolar "las relaciones interpersonal e interprofesional con el estudiante", Mertz (2004) destaca las funciones: (a) de desarrollo psicosocial, con la que el tutor facilita apoyo social y emocional al aprendiz; (b) de desarrollo profesional, cuando el tutor utiliza su conocimiento para ayudar al aprendiz a acercarse a lo que necesita para la toma de decisiones, mejorar su actuación y desarrollarse profesionalmente. En las distintas propuestas y estudios sobre la tutoría destacan las funciones psicosociales de apoyo, de establecimiento de un clima y relación de confianza adecuados, además de las de análisis, comprensión y reflexión sobre el ejercicio de la profesión. El asesoramiento y la competencia de relación interprofesional, comprenden la observación y la evaluación necesarias para un apoyo pertinente en la zona de desarrollo próximo del aprendiz.

Apenas se encuentran referencias sobre la función evaluadora del tutor escolar en la bibliografía sobre el acompañamiento, aunque en la realidad se le requiera. En un cierto sentido, la función evaluadora podría contradecir el establecimiento de ese nexo de confianza mediante la resolución de las tensiones propias de la complejidad del ejercicio docente facilitadas por experto. Aun así, desde el punto de vista formativo la participación del tutor escolar en la evaluación es necesaria porque ofrece una visión experta en la adquisición de las competencias.

La evaluación de competencias supone, en relación a la evaluación tradicional, una complejidad superior en la verificación de su adquisición. Algunos autores (Hall y Burke, 
2004; Kaftan, Buck y Haack, 2006) enfatizan su función [in]formativa para el estudiante, sobre la movilización eficiente de recursos internos y externos para resolver situaciones reales. Las competencias descritas en el plan de estudios a partir de las prescripciones ministeriales (orden ECI/3857/2007) ${ }^{4}$ requieren su explicitación, ${ }^{5}$ el estudio de su contenido y su vinculación a lo que sucede en el aula para evitar un tratamiento virtual (Jonnaert, 2002). Junto a su contenido, interesa dar a conocer los niveles de dominio, los agentes evaluadores y los instrumentos de evaluación.

Entendemos que la evaluación formativa es uno de los mayores retos en educación superior, como estrategia que informa al estudiante y al sistema, a la vez que permite implementar cambios que supongan mejoras en los procesos personales e institucionales generadores de mejoras en los procesos de enseñanza. La utilización de diversos instrumentos para sistematizar los procesos de observación y recogida de información parece fundamental para acercarnos al desempeño de la competencia en escenarios reales. Una de las principales dificultades se sitúa en la realización de los juicios de valor, derivados de la observación del desempeño de la competencia. Es necesario que los diferentes agentes que participan en el proceso de evaluación sean conocedores de su finalidad y funciones, de su posicionamiento ante ella, así como del uso de los instrumentos.

\section{CONTEXTO, OBJETIVO Y MÉTODO}

Con el curso 2012-2013 comienza en la Universidad de Lleida (Cataluña, España) una experiencia de formación dual de maestros, con una presencialidad distribuida de los estudiantes en dos contextos formativos, universidad y escuela de educación primaria, en una proporción del 60 y 40 por ciento respectivamente. Se trata de una iniciativa que intensifica la inmersión en el escenario profesional como estrategia para la adquisición y desarrollo de las competencias docentes. En la actualidad, la experiencia abraza dos cohortes, esperando la instauración plena para los cuatro años del Grado de Educación Primaria en el curso 2015-2016.

La existencia de las dos realidades formativas requirió un dispositivo de integración de aprendizajes para promover el ejercicio de relación, favorecer la intersección de las experiencias, reducir y aprovechar las tensiones derivadas de la actividad en ambos escenarios. Con este fin se diseñó la materia Integración I, con una evaluación compartida entre los tutores de facultad y los tutores escolares. El programa de la materia se define como semiabierto. Bajo algunos planteamientos generales como el conocimiento de las competencias del plan de estudios (orden ECI/3857/2007), las bases de la observación en el aula (Anguera, 1988) y los gestos profesionales (Brudermann y Pélissier, 2008), los aprendices editaron su portafolio electrónico (Coiduras, Carrera y Jové, 2012) y se llevaron a cabo distintas dinámicas en la facultad, de gran grupo o grupo medio, de análisis de sus experiencias. Los tutores de centro evaluaron en dos informes el progreso de los aprendices en la actividad de centro, estos últimos además fueron observados individualmente en el

Orden ECI/3857/2007 del 27 de diciembre por la que se establecen los requisitos para la verificación de los títulos universitarios oficiales que habiliten para el ejercicio de la profesión de Maestro en Educación Primaria. Recuperado desde http://www.boe.es/boe/dias/2007/12/29/pdfs/A53747-53750.pdf el 20 de mayo de 2014. http://www.boe.es/boe/ dias/2007/12/29/pdfs/A53747-53750.pdf

5 Compartimos el punto de vista de Angulo y Redón (2011) sobre la confusión de la orden ministerial al equiparar objetivos y competencias. 
desarrollo de una actividad docente en el aula, diseñada previamente con su tutor escolar. En la coordinación entre tutores escolares (65) y de la facultad (3) se realizaron tres encuentros de coordinación del proceso, de evaluación de los estudiantes y de valoración de la primera edición de esta modalidad formativa respectivamente.

El objetivo de este estudio de caso es determinar y estudiar las diferencias entre las evaluaciones del profesorado universitario y los tutores de los centros escolares con funciones de supervisión y acompañamiento en una experiencia de formación dual de futuros maestros. Se elige el estudio de caso por considerarse una estrategia de investigación adecuada para comprender las dinámicas presentes en contextos singulares (Eisenhardt, 1989). En él pueden combinarse distintos métodos de recogida de información e interpretación con el fin de describir, verificar o generar teoría. También, se caracteriza como particularista, descriptivo, heurístico, inductivo, lo que resalta la importancia del foco de investigación como sistema integrado: una persona, un proceso, una institución o un grupo social. Yin (1993) subraya su utilidad para la investigación, revelación y comunicación a la comunidad científica de situaciones que no hubieran podido conocerse de otra forma. Este estudio se inscribe en lo que se ha llamado investigación evaluativa (McMillan y Schumacher, 2007; Stake, 2005), y está centrado en un contexto concreto de formación dual durante el año académico 2012-2013.

En el estudio contrastamos distintas proposiciones teóricas (Martínez, 2006) que pueden explicar la diferencia en las evaluaciones y la preferencia de los estudiantes por una evaluación más centrada en su actividad escolar: (a) los distintos roles formativos del profesorado universitario y de los maestras y maestros como tutores en los centros escolares pueden condicionar la mirada sobre el aprendizaje del estudiante en formación en alternancia, y también la evaluación misma. Especialmente, el apoyo social y moral (Mertz, 2004), el ofrecimiento de una amistad profesional (Shea, 1992), el tutor como facilitador del desarrollo personal (Molina, 2008), son aspectos que podrían influenciar en la evaluación. (b) el estudiante suele reconocer en el docente del centro este acompañamiento personal estrechamente vinculado a la acción del aula como el más beneficioso en su formación y evaluación (Gutiérrez et al., 2011). La perspectiva de la actuación de unos y otros también puede ser distinta en función del foco de su acompañamiento. Mientras los tutores universitarios analizan la parte más reflexiva, los tutores de centro buscan que los alumnos sean más resolutivos en la acción. Aunque desde la universidad se aprecie la contribución desde la escuela al análisis y la comprensión de las situaciones, en los centros no suele disponerse del tiempo necesario para ello. Por otra parte, el profesorado universitario, alejado a menudo de la realidad escolar, podría dejar en un segundo plano la acción propiamente docente en el aula (Marcelo y Estebaranz, 1998).

Con la intención de reducir los sesgos se utilizan múltiples fuentes de información y evidencias (Figura 1 y Tabla 2) en torno a las percepciones y evaluación, para conseguir la complementariedad, aprovechar las fortalezas y compensar las debilidades de las evidencias. Como fuentes de información se contó con los resultados de las encuestas de la Oficina de Calidad $^{6}$, del sistema de garantía interna de la propia universidad, administradas por agentes externos, los informes de evaluación de los tutores escolares y los de los tutores de la facultad.

Con certificación de la Agencia de Calidad Universitaria de Cataluña (AQU) en base a los parámetros homogéneos de las otras agencias del estado español, de acuerdo con los Standards and Guidelines for Quality Assurance in the European Higher Education, validados por los distintos estados de la Unión Europea. 
Figura 1. Fuentes de información

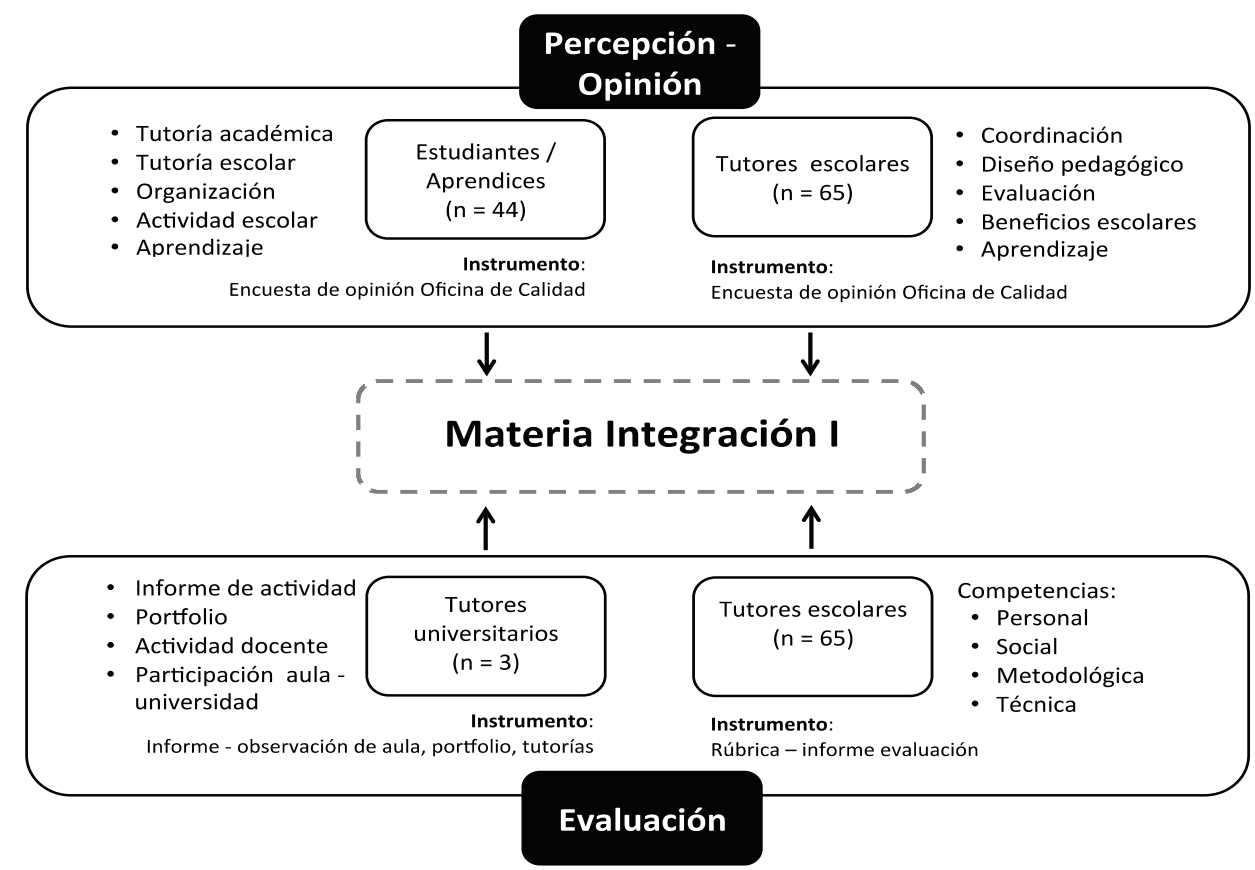

Tabla 2. Análisis de la información

\begin{tabular}{|l|c|c|c|c|c|}
\hline Actores & Estudiantes & \multicolumn{2}{|c|}{ Tutores escolares } & \multicolumn{2}{|c|}{ Tutores universitarios } \\
\hline $\begin{array}{l}\text { Fuente de } \\
\text { información }\end{array}$ & $\begin{array}{c}\text { Encuesta de } \\
\text { opinión } \\
\text { Anexo 1 }\end{array}$ & $\begin{array}{c}\text { Encuesta de } \\
\text { opinión } \\
\text { Anexo 2 }\end{array}$ & $\begin{array}{c}\text { Evaluación } \\
\text { curso } \\
\text { Anexo 3 }\end{array}$ & $\begin{array}{c}\text { Observación } \\
\text { de aula } \\
\text { Anexo 4 }\end{array}$ & $\begin{array}{c}\text { Evaluación } \\
\text { curso }\end{array}$ \\
\hline $\begin{array}{l}\text { Estadística } \\
\text { descriptiva }\end{array}$ & $\bullet$ & $\bullet$ & $\bullet$ & $\bullet$ & $\bullet$ \\
\hline $\begin{array}{l}\text { Análisis de } \\
\text { contenido }\end{array}$ & $\bullet$ & $\bullet$ & $\bullet$ & & \\
\hline
\end{tabular}

Con las encuestas de opinión la universidad pretende obtener información para la evaluación de la docencia en las distintas materias, logrando en este caso la opinión de los aprendices (Anexo 1) y de sus tutores en el centro escolar (Anexo 2). En los informes de evaluación de la materia integración, el tutor escolar responde a un conjunto de afirmaciones, basadas en la tipología de Bunk (1994) (Anexo 3). Los tutores universitarios otorgan una calificación numérica que sintetiza el rendimiento en distintas actividades, evidencias en el e-portafolio y en la observación del aprendiz conduciendo una actividad con un grupo de alumnos de educación primaria (Anexo 4). El documento para la observación de aula es la adaptación de una pauta de observación de la administración educativa en Catalunya de los maestros noveles en su primer año de ejercicio. 
Estudios Pedagógicos, vol. XL, Número Especial 1: 29-48, 2014

LA EVALUACIÓN DE COMPETENCIAS EN UNA EXPERIENCIA DE FORMACIÓN DUAL DE MAESTROS:

DIFERENCIAS Y SEMEJANZAS ENTRE TUTORES DE ESCUELA Y DE UNIVERSIDAD

El análisis de la información de las encuestas y los informes combina (Tabla 2) el análisis desde la estadística descriptiva y el análisis de contenido.

\section{PRESENTACIÓN DE RESULTADOS Y DISCUSIÓN}

De forma introductoria, parece necesario presentar un alto nivel de satisfacción, tanto de los estudiantes como de los tutores escolares, sobre la experiencia de la formación en alternancia. Ante la afirmación "Mi valoración global de la experiencia es buena", en la escala de Likert (1-5) unos y otros muestran un alto grado de acuerdo: de 4,20 y de 4,43 respectivamente.

Gráfico 1. Calificaciones de la materia de integración

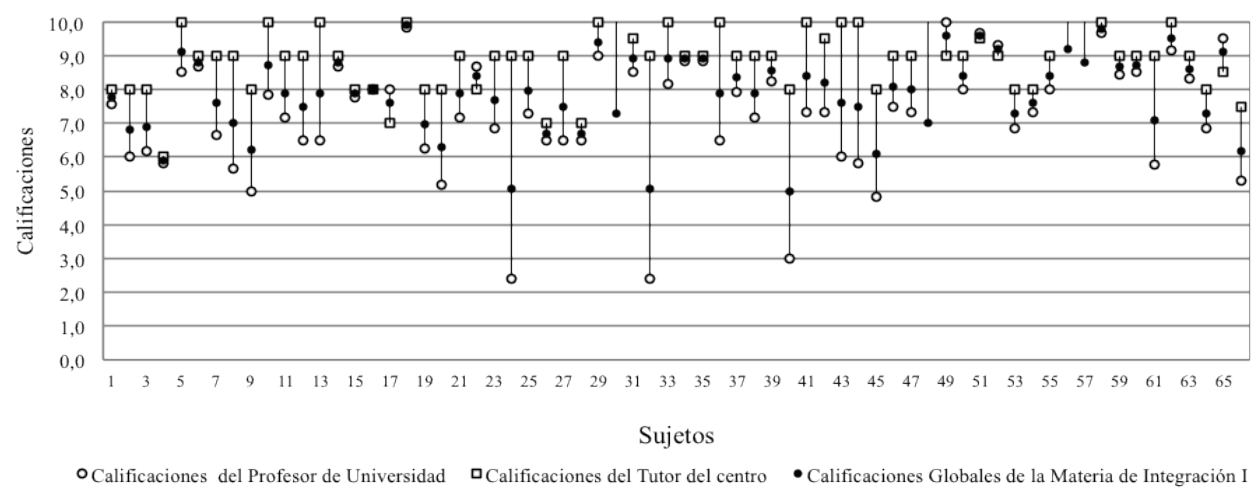

Las calificaciones de la materia de integración (Gráfico 1) en la actividad escolar evaluada por el tutor del centro escolar $(\square)$ y en la actividad universitaria evaluada por el tutor de universitario $(O)$ muestran una diferencia media de 1,2 puntos $(\bar{X}=8,8$ tutor escolar y $\bar{X}=7,6$ tutor universitario). Solamente un $44 \%$ de los aprendices (29 estudiantes) obtiene calificaciones similares (con diferencias no superiores a un punto) en las valoraciones atribuidas por los tutores del centro y los tutores de la universidad, con una baja dispersión cercana a la $\bar{X}(\bullet)$. Entre estos aprendices con dispersión menor en las cualificaciones se encuentran las tres mejores calificaciones. Sucede todo lo contrario en las calificaciones más bajas, donde se aprecia la máxima diferencia entre ellas, lejanas entre el tutor de centro $(86 \%>8)$ y el tutor de facultad $(36 \%>8)$.

La distinta naturaleza de las actividades que se desarrollan en ambos escenarios, no impide que nos cuestionemos sobre esa discrepancia que en algunos casos, como se observa en el Gráfico 1, llega a los 6 puntos. Algunas explicaciones podrían relacionarse con la perspectiva formativa y la relación de acompañamiento que ambos cotutores, tutor de escuela y tutor de universidad, establecen con el aprendiz en el curso de sus experiencias. La actividad solicitada en la universidad está sobre todo centrada en el análisis y la comprensión de la actividad escolar, de las propias actuaciones, del aprendizaje, de la gestión del aula, de la evaluación, de la utilización de recursos y estrategias pedagógicas, así como de la relación y movilización de los marcos teóricos. En la escuela prevalece la participación y la acción en 
el aula con los alumnos en colaboración con el mismo tutor en las distintas tareas docentes. Podría tratarse de una diferencia de prioridades en la formación inicial, según el rol del tutor.

Mientras la universidad prioriza la reflexión y el análisis de la práctica profesional en situaciones grupales, la escuela se centra en las actuaciones en situaciones reales y prácticas (Cuban, 1992). En la facultad los tutores suelen centrarse en aspectos como las escenas observadas, el análisis de la implementación de las actividades y los procesos de enseñanza aprendizaje, la evaluación y concreción curricular, las características del alumnado -desde una orientación generalmente teórica- para determinar que un aprendiz moviliza de forma holística sus competencias profesionales (Castejón Oliva et al., 2011). Estas diferentes perspectivas y lógicas formativas (Gutiérrez et al., 2011) proporcionan una posible explicación de las calificaciones inferiores de los tutores universitarios respecto a las de los tutores escolares. El tutor de universidad suele dirigir una secuencia estructurada que relacionamos directamente con un propósito de relación con los sistemas conceptuales. En la escuela, la proximidad y la relación uno a uno compartiendo el rol docente, aún en la distancia de la experiencia y experticia, suele establecer una relación de complicidad y de ayuda más cercana y personal. En la encuesta de opinión de la Oficina de Calidad, escala de Likert, los estudiantes juzgan como adecuadas las actuaciones del tutor escolar para hacer posible el inicio en las tareas y práctica de las habilidades profesionales $(\bar{X}=4,11$, ítem 12), orientando su actuación a la consecución de los objetivos formativos ( $\bar{X}=4,18$, ítem 5). En cambio, su satisfacción con el acompañamiento que le ofrece el tutor universitario es inferior, con una valoración media de 3,70 ante la afirmación "El tutor académico muestra interés en la tutorización" (ítem 1). Esta diferencia, de 0,5 puntos, podría interpretarse como una cierta preferencia a la actuación del primero respecto a la actuación del segundo.

Los estudiantes valoran la comunicación entre los tutores como baja (ítem 7), con un grado de acuerdo de 2,20 ante la afirmación "Hay coordinación entre los dos tutores". En sus comentarios explican su percepción sobre la comunicación entre tutores:

La coordinación entre universidad y escuelas es mejorable, además como hemos comentado entre nosotros las realidades entre las distintas escuelas y las visiones de los tutores escolares son muy distintas. Sin coordinación, la experiencia se convierte sobre todo en una experiencia de autoaprendizaje. Estudiante 12.

Se ha caracterizado la función del tutor escolar como de implicación en una relación de ayuda respecto al estudiante. Su labor es potenciar -desde una relación de afecto- el crecimiento personal y profesional (Siles et al., 2004). La relación que se establece se explica también por un apoyo mutuo. El estudiante en su aprendizaje en el aula como docente en formación colabora activamente, bajo las indicaciones de su mentor, en el desarrollo de las experiencias de enseñanza adquiriendo, paulatinamente, una disposición más proactiva en la toma de decisiones sin necesidad de consultarlas. En este punto el tutor escolar visualiza una contribución útil que en la mayor parte de los casos mejora la actividad de enseñanza consistente en una atención más personalizada, la atención a la diversidad, el análisis y la corrección de las producciones del alumnado, la mejora del clima del aula, etc. En este sentido, los tutores escolares muestran en la encuesta de opinión, escala de Likert (1-5), un alto grado de acuerdo $(\bar{X}=4,58)$ con la afirmación "La presencia del estudiante en el lugar de trabajo resulta positiva para el desarrollo de la actividad profesional del centro". También indican una percepción muy positiva sobre la actitud hacia el aprendizaje de los estudiantes $(\bar{X}=4,60)$ : "Los estudiantes han demostrado disponibilidad para el aprendizaje". Algunos comentarios de los tutores se realizan en la misma dirección: 
Estudios Pedagógicos, vol. XL, Número Especial 1: 29-48, 2014

LA EVALUACIÓN DE COMPETENCIAS EN UNA EXPERIENCIA DE FORMACIÓN DUAL DE MAESTROS:

DIFERENCIAS Y SEMEJANZAS ENTRE TUTORES DE ESCUELA Y DE UNIVERSIDAD

En nuestro centro hemos constatado su disponibilidad para colaborar en las tareas escolares: atendiendo a grupos reducidos de alumnos, conduciendo a veces tareas concretas, ... e incluso, en ocasiones, colaborando en el desarrollo de actividades escolares fuera del horario comprometido con el centro. Tutor Escolar [TE].

La formación ha sido paralela, se ha producido en ambos sentidos, también en nosotros los tutores. Su presencia en el centro ha favorecido el desarrollo de la actividad docente ya que, en general, han mostrado una gran participación. TE 45.

En general, ya en el primer curso, se da la vivencia del aprendiz de maestro como un colaborador participante, como agente de mejora que proporciona un beneficio en términos amplios sobre el desempeño de la enseñanza. Este pensamiento profesional explicaría, en parte, una evaluación alta de los aprendices que tutoran.

Cuando los tutores escolares evalúan a los estudiantes, se observa un agrupamiento de las calificaciones entre los valores 3 y 4 (escala 1-4). En el detalle de la evaluación de las cuatro competencias (técnica, metodológica, social y participativa, según la tipología de Bunk, 1994), evaluadas a partir del conjunto de indicadores del informe de curso de los tutores escolares (Anexo 3), se comprueba que las diferencias entre alumnos son bajas (gráfico 2).

Gráfico 2. Comparación de calificaciones más altas y más bajas respecto a $\bar{X}$ en el informe del tutor de escuela

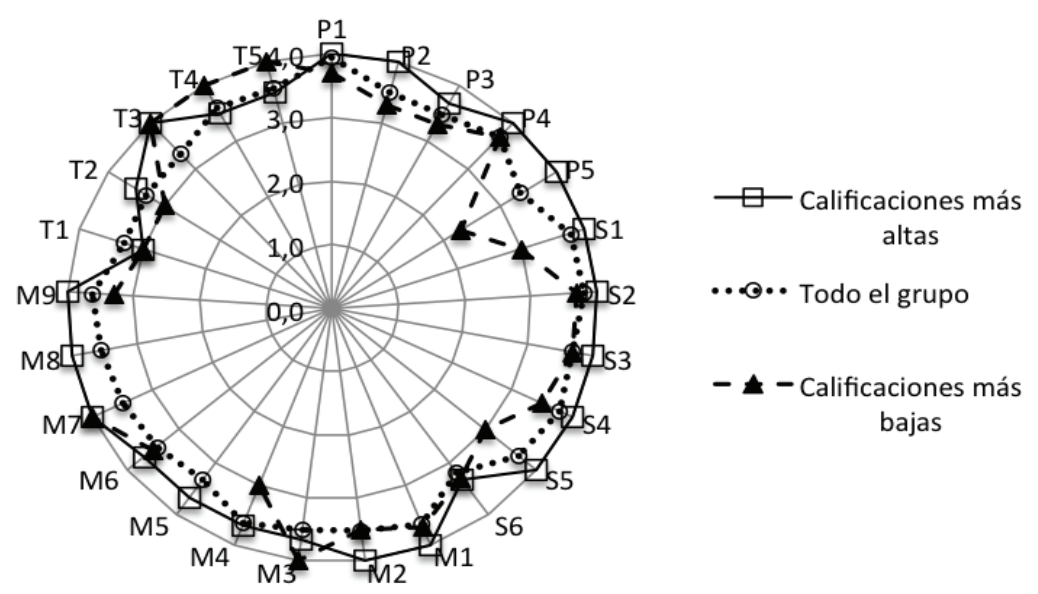

Cuando se analiza la evaluación del tutor universitario y del tutor escolar en función del género de los estudiantes se observa que en ambas calificaciones las mujeres puntúan más alto. En el caso de los hombres, han obtenido un promedio de 6,65 en la calificación de los tutores de la universidad, frente al 7,9 de las mujeres (diferencia entre promedios 1,25). En las valoraciones atribuidas por los tutores escolares, los hombres obtienen un promedio de 8,53 frente al 8,9 de las mujeres (diferencia entre promedios 0,37). Resulta también relevante el siguiente dato: un $6 \%$ de las mujeres y un $4 \%$ de los hombres han obtenido calificaciones inferiores a 4 en la valoración del tutor de universidad; mientras que la calificación menor atribuida por los tutores de los centros es de 6 (diferencia entre promedios 2). 
Para la evaluación de la competencia metodológica, desde la universidad se realizó una observación de aula (Anexo 4) donde los aprendices conducían la clase en una actividad guiada, diseñada con la supervisión del tutor escolar. Por otro lado, el tutor escolar valoró la competencia metodológica en su informe de evaluación. De la pauta de observación de aula y del informe escolar sobre la competencia metodológica se han agrupado las valoraciones por su similitud conceptual (Tabla 3) en: (1) Gestión y Organización de la actividad y del aula. Clima en proceso de enseñanza/aprendizaje. (2) Comunicación con el alumno: expresión adecuada al registro e interacción con los alumnos. (3) Desarrollo de la intervención: organización pedagógica en el aula, promoción de actividades para adquirir los objetivos con un ritmo adaptado a las necesidades de los alumnos, utilización de estrategias pedagógicas para el desarrollo de las actividades y evaluación del proceso de enseñanza/aprendizaje, y (4) Utilización de recursos, medios TIC, etc.

Tabla 3. Síntesis de criterios de evaluación de la competencia metodológica

\begin{tabular}{|c|c|c|c|c|}
\hline $\begin{array}{c}\text { Criterios de la Observación Guiada } \\
\text { Tutor Universitario }\end{array}$ & $\bar{X}$ & $\begin{array}{l}\text { Criterios de evaluación de la } \\
\text { competencia metodológica }\end{array}$ & $\bar{X}$ & $\begin{array}{l}\text { Criterios del Informe escolar. Tutor } \\
\text { escolar. }\end{array}$ \\
\hline $\begin{array}{l}\text { 1.1. Presenta la actividad de forma clara } \\
\text { y el qué se pretende con ella } \\
\text { 1.2. Organiza el tiempo para desarrollar } \\
\text { la actividad prevista } \\
\text { 1.3. Dinamiza el aula favoreciendo un } \\
\text { clima de trabajo y de respeto } \\
\text { 1.4. Se muestra seguro y tranquilo } \\
\text { 2.2.2. Refuerza y motiva positivamente } \\
\text { a los alumnos } \\
\text { 2.4.2. Promueve la participación }\end{array}$ & 3,10 & $\begin{array}{l}\text { 1. Gestión y Organización de } \\
\text { la actividad y del aula: } \\
\text { clima adecuado para el proceso } \\
\text { de enseñanza/aprendizaje }\end{array}$ & 3,3 & $\begin{array}{l}\text { 1. Es sensible y responde con ayuda a la } \\
\text { necesidades de los alumnos } \\
8 \text {. Establece un clima propicio en la } \\
\text { actividad de enseñanza y aprendizaje }\end{array}$ \\
\hline $\begin{array}{l}\text { 2.1.1. Se expresa con un registro } \\
\text { docente adecuado a la edad del } \\
\text { alumnado } \\
\text { 2.1.2. Utiliza el léxico adecuado en la } \\
\text { actividad y los contenidos } \\
\text { 2.1.3. Apoya la comunicación verbal } \\
\text { con la no verbal } \\
\text { 2.2.1. Correcta interacción y contacto } \\
\text { con los alumnos }\end{array}$ & 3,15 & $\begin{array}{l}\text { 2. Comunicación con el } \\
\text { alumno y al grupo: } \\
\text { expresión adecuado al registro e } \\
\text { interacción positiva con los } \\
\text { alumnos }\end{array}$ & 3,3 & $\begin{array}{l}\text { 4. Se muestra abierto a les cuestiones } \\
\text { que el alumnado planeta } \\
\text { 9. Refuerza y motiva positivamente a los } \\
\text { alumnos }\end{array}$ \\
\hline $\begin{array}{l}\text { 2.3.1. La actividad es adecuada a los } \\
\text { conocimientos previos } \\
\text { 2.3.2. El ritmo de la actividad es } \\
\text { adecuado } \\
\text { 2.3.3. Las dinámica promueve la } \\
\text { actividad del alumnado } \\
\text { 2.3.4. Hace intervenciones oportunas } \\
\text { para promover la progresión en la } \\
\text { actividad } \\
\text { 2.4.1. Utiliza ejemplos para potenciar la } \\
\text { comprensión y el aprendizaje } \\
\text { 2.4.3. Se muestra creativo en el } \\
\text { desarrollo de la actividad favoreciendo } \\
\text { el aprendizaje } \\
\text { 3.1. Potencia en el alumnado la revisión } \\
\text { para la corrección de su actividad } \\
\text { 3.2. El alumnado sabe aquello que } \\
\text { realiza correctamente y los aspectos } \\
\text { que tiene que mejorar }\end{array}$ & (1) & $\begin{array}{c}\text { 3. Desarrollo de la } \\
\text { intervención: } \\
\text { organización pedagógica en el } \\
\text { aula, promoción de actividades } \\
\text { para adquirir los objetivos con } \\
\text { un ritmo adaptado a las } \\
\text { necesidades de los alumnos, } \\
\text { utilización de estrategias } \\
\text { pedagógicas para el desarrollo } \\
\text { de las actividades y evaluación } \\
\text { del proceso de enseñanza/ } \\
\text { aprendizaje }\end{array}$ & 3,2 & $\begin{array}{l}\text { 2. Actúa en base al conocimiento del } \\
\text { grupo y del proyecto educativo } \\
\text { 3. Las actividades pedagógicas son } \\
\text { coherentes con los objetivos educativos } \\
\text { 5. Promueve en el alumnado la relación } \\
\text { de los contenidos nuevos con otros ya } \\
\text { trabajados }\end{array}$ \\
\hline $\begin{array}{l}\text { 4.1. Utiliza objetos, materiales, } \\
\text { situaciones reales para reforzar su } \\
\text { exposición }\end{array}$ & 3,3 & $\begin{array}{l}\text { 4. Utilización de recursos, } \\
\text { medios TIC, etc. }\end{array}$ & 3,5 & $\begin{array}{l}\text { 6. Utiliza, de forma pertinente, diferentes } \\
\text { lenguajes (gráfico, textual, AV, TIC) } \\
\text { 7. Utiliza recursos adecuados para las } \\
\text { actividades de enseñanza y aprendizaje }\end{array}$ \\
\hline
\end{tabular}


En una escala sobre 4, los resultados (Gráfico 3) muestran valoraciones superiores a 3.00, con una gran semejanza entre las valoraciones emitidas por los tutores escolares y los tutores de la universidad. Esta alta coincidencia entre evaluadores, en relación a la competencia metodológica, llama la atención. Por un lado, la colaboración en el aula con el tutor a lo largo del curso, en una especie de colegialidad, podría explicar en parte la valoración alta desde la escuela de la competencia metodológica. Por otro, el tutor universitario realiza observaciones que se han previsto en el tiempo, que han podido ser ensayadas y que cuentan, el día de su realización y en la mayor parte de los casos, con la complicidad de los alumnos de educación primaria, como se ha comprobado después de 120 observaciones realizadas en distintos momentos.

Gráfico 3. Comparación entre la evaluación (tutor de centro escolar) y la observación (tutor facultad) en la competencia metodológica

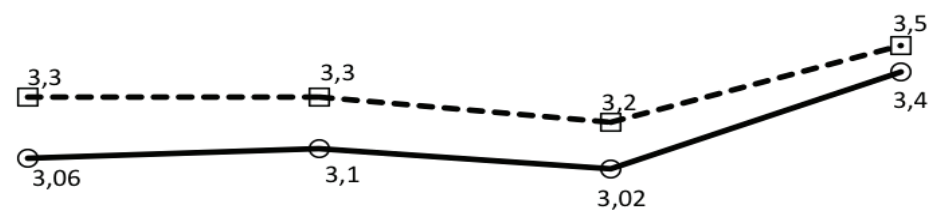

1

La experiencia de la observación en el aula como una actividad programada y objeto de evaluación de la materia Integración I ha sido reconocida como un estímulo eficaz para los tutores escolares y los mismos aprendices. Los tutores escolares han animado al aprendiz a la acción y conducción del grupo de alumnos de educación primaria en distintos momentos, conscientes de la necesidad del ensayo. Para el aprendiz la observación ha motivado, más allá de la observación, su colaboración y práctica docente.

\section{CONCLUSIONES}

Después de los resultados del estudio de caso puede afirmarse, en consonancia con las proposiciones teóricas, que la evaluación de la experiencia escolar del estudiante está relacionada con el foco, la mirada y la posición del evaluador. Las diferencias entre evaluaciones por ambos tutores, que se constatan en la evaluación general, pueden explicarse en base a las actividades y demandas distintas que se realizan en los dos contextos, el rol como tutor y las funciones desde la escuela y la universidad.

Distintos autores (Mertz, 2004; Molina, 2008; Shea, 1992) han puesto de relieve la cercanía del tutor escolar al aprendiz compartiendo el desarrollo de la actividad de aula. La función de acompañamiento psicosocial y de ayuda emocional en el centro escolar puede condicionar valoraciones altas. Las altas puntuaciones, vistas en su conjunto, tienen un cierto paralelismo con la alta valoración sobre la experiencia de formación dual. La 
expresión de esta satisfactoriedad está en la línea con las apreciaciones de Hoeckel (2008), cuando identifica una experiencia en beneficio mutuo, de colaboración con una incidencia positiva tanto en el funcionamiento de la clase y aprendizaje del alumnado de educación primaria como en la formación inicial para la docencia.

En el pensamiento de los estudiantes, la actividad escolar tiene una cierta supremacía sobre la universitaria. Desde esta idea ponen en valor su implicación en el día a día del aula y reclaman la traducción de su participación en la evaluación, con un mayor protagonismo del tutor escolar. Los distintos actores de la experiencia reconocen esta actuación, lo que difiere de otras constataciones en estudios sobre el prácticum (Guerrero y López, 2006) donde un elevado número de estudiantes manifiesta un bajo grado de participación y autonomía en las actividades docentes.

Cabe pensar que si las diferencias en la evaluación responden a distintas lógicas formativas de los tutores, escolares y universitarios, o a las iniciativas que favorecen la relación de las actividades de ambos entornos, estas discrepancias pueden afrontarse en su potencialidad formativa. Una de las vías que proponemos consiste en la revisión de las estrategias y dispositivos de integración. Por un lado en el contraste de los conocimientos con la práctica, y por otro en el estudio de la sincronía entre las temáticas tratadas en la universidad y las experiencias de acompañamiento en el centro escolar. El contraste de teoría y práctica, se aborda acercando los marcos teóricos y las disposiciones que regulan el sistema a realidades de aula y centro concretas. $\mathrm{Y}$ en sentido inverso, incidir en actividades en al aula de educación primaria con potencialidad formativa es posible por la vía de la acción y como motivación del análisis, la interpretación, la argumentación y el desarrollo de un juicio práctico (Correa Molina y Gervais, 2011; Fenstermacher, 1994, 1996). Los ajustes temporales, en algunos momentos facilitando la sincronía, favorecerán un análisis simultáneo en los centros escolares y en la universidad. El referente de las competencias básicas de educación primaria desde las didácticas específicas y los fundamentos psicopedagógicos de la profesión puede formar parte del acuerdo para los diseños curriculares, su implementación posterior en el aula, motivo de apoyo, seguimiento, observación y análisis.

El rol de los tutores universitario y escolar está mediatizado por la actividad en el contexto y parece innecesario forzar su ejercicio en otro sentido. Pero sí que su desarrollo pueda evolucionar por la vía de la formación conjunta, como un modo de acompañamiento de la experiencia basado en el análisis de la práctica. El desarrollo de seminarios de análisis de la actividad del aprendiz y la revisión de las estrategias de tutoría tiene el potencial de incidir sobre el conocimiento de las funciones y el análisis de su ejercicio, el consenso sobre los significados de los aspectos claves de la formación, el desarrollo profesional conjunto y la mejora de la función evaluadora. Disminuir la distancia entre las visiones de ambos tutores requiere, por un lado, acercar al profesor universitario al ejercicio de la docencia en la escuela y, por otro, acercar al docente escolar a las funciones propias de tutor para las que no recibió formación. No somos partidarios de una diferenciación excesiva de los roles que resultaría artificial; sino más bien de compartir, admitiendo las miradas distintas, el análisis, la reflexión y el acercamiento a la realidad y a la acción en el aula (Marcelo y Estebaranz, 1998) como una forma de avanzar hacia una formación dual de lógica integrativa.

La evaluación de la competencia metodológica en este estudio, aun teniendo en cuenta la gran coincidencia en las valoraciones, puede presentar alguna limitación derivada del mismo instrumento así como de la diversidad posible de interpretaciones por cada uno de los tutores escolares y de universidad. El uso de rúbricas validadas (Danielson, 2013; Good 
Estudios Pedagógicos, vol. XL, Número Especial 1: 29-48, 2014

LA EVALUACIÓN DE COMPETENCIAS EN UNA EXPERIENCIA DE FORMACIÓN DUAL DE MAESTROS:

DIFERENCIAS Y SEMEJANZAS ENTRE TUTORES DE ESCUELA Y DE UNIVERSIDAD

et al., 2006; Tsang-Hester, 2003) nos parece un camino necesario a recorrer, no solamente por sus posibilidades evaluativas, sino también por su potencialidad formativa para los tutores y los mismos estudiantes. La utilización de estas herramientas no puede suplir los acuerdos que deben darse entre tutores escolares y de universidad sobre las competencias que se observan y evalúan, sobre la potencialidad formativa de las actividades escolares, en el aula universitaria y su evaluación.

\section{REFERENCIAS BIBLIOGRÁFICAS}

American Council on Education. (1999). To Touch the Future: Transforming the Way Teachers Are Taught. Washington, DC: US. An Action Agenda for College and University Presidents.

Anguera, M.T. (1988). L'observació a l' escola. Barcelona: Graó.

Angulo, R. y Redon, S. (2011). Competencias y contenidos: cada uno en su sitio en la formación docente. Estudios Pedagógicos, vol.37, n.2, 281-299.

Armengol, C., Rodríguez-Gómez, D., Gairín, J., del Arco, I. y Muñoz, J.L. (2013). Un modelo de intervención para la organización y el desarrollo de las prácticas curriculares en la universidad. En P.C. Muñoz, M. Raposo-Rivas, M. González, M.E. Martínez-Figueira, M. Zabalaza-Cerdeiriña, y A. Pérez-Abellás, Un Practicum para la formación integral de los estudiantes (pp. 1-12). Santiago de Compostela: Andavira.

Brudermann, C., et Pélisser, C. (2008). Les gestes professionnels de l'enseignant: une analyse pédagogique et une représentation informatisée pour la formation-L'exemple des langues étrangères. Revue internationale des technologies en pédagogie universitaire, vol.5, n.2, 21-33.

Bunk, G.P. (1994). La transmisión de las competencias en la formación y perfeccionamiento profesionales de la R.F.A. Revista Europea de Formación Profesional, n.1, 8-14.

Castejón, F.J., López-Pastor, V.M., Julián, J.A. y Zaragoza, J. (2011). Evaluación formativa y rendimiento académico en la formación inicial del profesorado de Educación Física. Revista Internacional de Medicina y Ciencias de la Actividad Física y el Deporte, vol.11, n.42, 328-346.

European Centre for the Development of Vocational Training. (2008). Terminology of European education and training policy. A selection of 100 key terms. Luxembourg: Office for Official Publications of the European Communities.

Coiduras, J.L., Carrera, X., et Jové, C.M. (2012). Le e-porfolio comme espace d'accompagnement et d'intégration des apprentissages dans des processus de formation en alternance. TransFormations. Recherches en Education et Formation des Adultes, n.7, 79-92.

Commission of the European Communities. (2007) Communication from the Commission to the Council and the European Parliament on Improving the Quality of Teacher Education, 2007. Brusselas: Unión Europea. Recuperado desde http://ec.europa.eu/education/com392_en.pdf

Correa Molina, E., et Gervais, C. (2011). Se former professionnellement par la pratique: une dynamique individuelle et collective. Revue des sciences de l'éducation, vol.37, n.2, 231-236.

Cuban, L. (1992). Managing dilemmas while building professional communities. Educational Researcher, vol.21, n.1, 4-11.

Danielson, C. (2013). The framework for teaching-2013 edition. Evaluation instrument. Princeton: USA. Recuperado desde: http://www.danielsongroup.org

Danielson, C. (2013). The Framework for Teaching. Princeton: The Danielson Group.

Eisenhardt, K.M. (1989). Building Theories from Case Study Research. Academy of Management Review, vol.14, n.4, 532-550.

Fenstermacher, G. (1994). The knower and the known. The nature of knowledge in research on teaching. In L. Darling-Hammond (Ed.), Review of Research On Education, 20 (pp. 3-56). Washington, D.C.: American Educational Research Association. 
Fenstermacher, G. (1996). Les arguments pratiques dans la transformation morale de l'enseignement d'une discipline. Revue des sciences de l'éducation, vol.22, n.3, 617-634.

Fernández Enguita, M., Mena, L. y Rivere, J. (2010). Fracaso abandono escolar en España (Colección Estudios Sociales, 29). Barcelona: La Caixa.

Good, T., McCaslin, M., Tsang, H., Zhang, J., Wiley, C., Bozack, A., \& Hester, W. (2006). How well do $1^{\text {st }}$-year teachers teach. Does type of preparation make a difference? Journal of Teacher Education, vol.57, n.4, 410-430.

Guerrero, M. y López, M. (2006). El prácticum en la formación de pedagogos ante la convergencia europea. Algunas reflexiones y propuestas de mejora. Revista de Educación, n.341, 517-552.

Gutiérrez, C., Pérez, A., Pérez, M. y Palacios, A. (2011). Percepciones de profesores y alumnos sobre la enseñanza, evaluación y desarrollo de competencias en estudios universitarios de formación de profesorado. Cultura y Educación: Revista de teoría, investigación y práctica, vol.23, n.4, 499-514.

Hall, K., \& Burke, W. (2004). Making formative assessment work: Effective practice in the primary classroom. England: McGraw-Hill International.

Hoeckel, K. (2008). Costs and Benefits in Vocational Education and Training. OCDE Report EDU/ EDPC/CERI-2008. Retrieved from: http://www.oecd.org/edu/skills-beyond-school/41538706.pdf

Jonnaert, P. (2002). Compétences et socioconstructivisme. Un cadre théorique. Bruxelles: De Boeck.

Kaftan, J., Buck, G., \& Haack, A. (2006). Using Formative Assessments to Individualize Instruction and Promote Learning. Middle School Journal, vol.37, n.4, 44-49.

Marcelo, C. y Estebaranz, A. (1998). Modelos de colaboración entre la Universidad y las Escuelas en la Formación del Profesorado. Revista de Educación, n.317, 97-120.

Martínez, M., y Albaigés, B. (Directors) (2013). L'estat de l'Educació a Catalunya. Anuari 2013. Barcelona: Fundació Jaume Bofill.

Martínez, M. (2006). El método de estudio de caso: estrategia metodológica de la investigación científica. Pensamiento \& Gestión, n.20, 165-193.

Maubant, P. (2009). La alternancia en formación, otra mirada para cuestionar los sentidos del análisis de las prácticas de enseñanza. Pensamiento Educativo. Revista De Investigación Educacional Latinoamericana, vol.44, n.1, 119-137.

McMillan, J.H. y Schumacher, S. (2007). Investigación educativa. Madrid: Pearson Educación.

Mertz, N.T. (2004). What's a Mentor, Anyway? Educational Administration Quarterly, vol.40, n. $4,541-560$.

Molina, E. (2008). Analysis of the system of Practicum in Spanish universities. European Journal of Teacher Education, vol.31, n.4, 339-366.

OCDE. (2012). Informe del Programa Internacional para la Evaluación de Estudiante. París: autor.

Portelance, L., Gervais, C., Lessard, M., et Beaulieu, P. (2008). La formation des enseignants associés et des superviseurs universitaires. Rapport de recherche. Québec: Ministère de l'Éducation, du Loisir et du Sport.

Shea, G.F. (1992). Mentoring. Londres: Kogan Page.

Siles, J., Cibanal, J., Castells, M., Domínguez, J., Gabaldón, E., Jurado, J., Núñez del Castillo, M., Pérez, R., Solano, M., Vila, M. y Vizcaya, M. (2004). Las diferencias culturales implicadas en el proceso de prácticas clínicas en enfermería. Una aportación desde la antropología de la complejidad y el pensamiento crítico. En G. Bernabeu y N. Sauleda (Eds.), Espacios de investigación en la profesionalización docente universitaria (pp. 97-116). Alicante: Universidad de Alicante.

Stake, R. (2005). Investigación con estudio de casos. Madrid: Morata.

Tsang-Hester, Y. (2003). Using standardized performance observations and interviews to assess the impact of teacher education (Unpublished doctoral dissertation, University of Arizona).

Yin, R.K. (1993). Applications of Case Study Research, Applied Social Research Methods (Series, vol. 34). Newbury Park, CA: Sage. 
Estudios Pedagógicos, vol. XL, Número Especial 1: 29-48, 2014

LA EVALUACIÓN DE COMPETENCIAS EN UNA EXPERIENCIA DE FORMACIÓN DUAL DE MAESTROS:

DIFERENCIAS Y SEMEJANZAS ENTRE TUTORES DE ESCUELA Y DE UNIVERSIDAD

\section{ANEXOS}

Anexo 1: Encuesta de opinión a los estudiantes

\section{Aspectos referidos al tutor/a académico/a}

Grado en que se presenta el indicador

1. El tutor académico muestra interés en la tutorización

2. Crea un clima de diálogo, confianza y trabajo

3. El seguimiento de mis experiencias por parte del tutor/a académico ha sido adecuada

4. Considero que el tutor/a académico/a es un buen tutor/a ................ $1 \begin{array}{lllll}1 & 2 & 3 & 4 & 5\end{array}$

\section{Aspectos referidos al tutor/a de la escuela}

5. El tutor de la escuela se ha preocupado para que alcanzara los objetivos del practicum

6. El tutor de la escuela ha creado las condiciones más relevantes para poder ensayar las habilidades más relevantes de la práctica profesional

\section{Aspectos referidos a la organización}

7. Hay coordinación entre los dos tutores (académico y empresa) .... $\begin{array}{llllll}1 & 2 & 3 & 4 & 5\end{array}$

8. El plan de trabajo acordado al inicio se ha seguido completamente

$\begin{array}{lllll}1 & 2 & 3 & 4 & 5\end{array}$

9. El procedimiento de evaluación es adecuado

\section{Aspectos referidos a la escuela}

10. La actividad está relacionada con mi formación

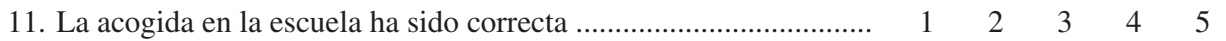

\section{Aspectos referidos al aprendizaje}

12. He podido practicar las habilidades profesionales en situaciones reales

13. He adquirido las competencias planteadas la materia

14. He sabido dar respuesta a las actuaciones que me han propuesto en el trabajo del centro

15. Mi valoración global de la experiencia es buena ......

$\begin{array}{ccccc}1 & 2 & 3 & 4 & 5 \\ 1 & 2 & 3 & 4 & 5 \\ 1 & 2 & 3 & 4 & 5 \\ 1 & 2 & 3 & 4 & 5\end{array}$

1. Completamente en desacuerdo 5. Completamente de acuerdo 
Anexo 2: Encuesta de opinión a los tutores de los centros escolares y resultados

\section{Aspectos referidos al tutor/a académico/a}

1. Los contactos con la universidad han sido suficientes para la organización del practicum

2. El diseño del programa (competencias, objetivos o actividades) es adecuado y sostenible

3. El procedimiento de evaluación es adecuado

4. La duración del practicum es suficiente

5. Los contactos con la universidad han sido suficientes para la organización

6. El diseño del programa (competencias, objetivos o actividades) es adecuado

7. El procedimiento de evaluación es adecuado

8. La duración del practicum es suficiente

9. La presencia del estudiante en el lugar de trabajo resulta positiva para el desarrollo de la actividad profesional del centro

10. Considero que estas prácticas, tal como están organizadas, ofrecen una buena posibilidad de aprendizaje profesional a los estudiantes

11. La formación previa de los estudiantes ha sido adecuada para el desarrollo de las prácticas

12. Los estudiantes han demostrado disponibilidad para el aprendizaje

13. Los estudiantes han conseguido los objetivos que se plantearon para las prácticas

14. Mi valoración global de la experiencia es buena

\section{Grado en que se} presenta el indicador

\begin{tabular}{lllll}
1 & 2 & 3 & 4 & 5 \\
1 & 2 & 3 & 4 & 5 \\
1 & 2 & 3 & 4 & 5 \\
1 & 2 & 3 & 4 & 5 \\
1 & 2 & 3 & 4 & 5 \\
1 & 2 & 3 & 4 & 5 \\
1 & 2 & 3 & 4 & 5 \\
1 & 2 & 3 & 4 & 5 \\
1 & 2 & 3 & 4 & 5 \\
1 & 2 & 3 & 4 & 5 \\
1 & 2 & 3 & 4 & 5 \\
1 & 2 & 3 & 4 & 5 \\
1 & 2 & 3 & 4 & 5 \\
1 & 2 & 3 & 4 & 5 \\
\hline
\end{tabular}

1. Completamente en desacuerdo 5. Completamente de acuerdo 
Estudios Pedagógicos, vol. XL, Número Especial 1: 29-48, 2014

LA EVALUACIÓN DE COMPETENCIAS EN UNA EXPERIENCIA DE FORMACIÓN DUAL DE MAESTROS:

DIFERENCIAS Y SEMEJANZAS ENTRE TUTORES DE ESCUELA Y DE UNIVERSIDAD

Anexo 3: Informe de evaluación del tutor de los centros escolares

Competencia personal [ saber ser ]

Grado en que se presenta el indicador

$\mathrm{P} 1$. Se manifiesta respetuoso con la comunidad educativa .......

nada Poco Bastante Mucho

P2. Tiene un comportamiento adulto con el alumnado

$\begin{array}{llll}1 & 2 & 3 & 4 \\ 1 & 2 & 3 & 4 \\ 1 & 2 & 3 & 4\end{array}$

P3. Se muestra seguro y tranquilo dentro del aula

$\begin{array}{llll}1 & 2 & 3 & 4\end{array}$

P4. Demuestra interés por la profesión y por la propia formación docente

P5. Reflexiona sobre su experiencia docente mostrando capacidad de autocrítica

$\begin{array}{llll}1 & 2 & 3 & 4\end{array}$

Comentarios:

Competencia social [ saber estar ]

S1. Es puntual, sigue el cronograma y se adapta a la normativa del centro

S2. Se muestra colaborador con el tutor, se compromete y participa en proyectos

S3. Participa en proyectos de centro colaborando con otros profesionales

S4. Cumple con los compromisos y el plan de trabajo acordado

S5. Mantiene con el tutor una comunicación pertinente sobre su formación

S6. Manifiesta iniciativa y hace propuestas

1

$\begin{array}{llll}1 & 2 & 3 & 4\end{array}$

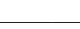

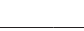

Comentarios:

Competencia metodológica [ saber hacer ]

M1. Es sensible y responde, con ayuda, a las necesidades del alumnado

M2. Actúa en base al conocimiento del grupo y del proyecto educativo

M3. Las actividades pedagógicas son coherentes con los objetivos educativos

M4. Se muestra abierto a las cuestiones que el alumnado plantea

M5. Promueve la relación de los contenidos nuevos con otros ya trabajados

M6. Utiliza, de forma pertinente, diferentes lenguajes (gráfico, textual, AV, TIC)

M7. Utiliza recursos adecuados para las actividades de enseñanza/ aprendizaje

M8. Establece un clima propicio en la actividad de enseñanza/ aprendizaje

M9. Refuerza y motiva positivamente a los alumnos

Comentarios: 
Estudios Pedagógicos, vol. XL, Número Especial 1: 29-48, 2014 LA EVALUACIÓN DE COMPETENCIAS EN UNA EXPERIENCIA DE FORMACIÓN DUAL DE MAESTROS: DIFERENCIAS Y SEMEJANZAS ENTRE TUTORES DE ESCUELA Y DE UNIVERSIDAD

\section{Competencia técnica [ saber ]}

T1. Reconoce en les actividades de aprendizaje el currículum de E. Primaria

$\begin{array}{llll}1 & 2 & 3 & 4\end{array}$

T2. Diseña actividades de aprendizaje adecuadas a las competencias básicas

$\begin{array}{llll}1 & 2 & 3 & 4\end{array}$

T3. Identifica los aspectos organizativos relacionados con las actividades

$\begin{array}{llll}1 & 2 & 3 & 4\end{array}$

T4. Se expresa con adecuación al registro docente

$\begin{array}{llll}1 & 2 & 3 & 4\end{array}$

T5. Domina los contenidos a impartir

$1 \quad 2 \quad 3$

4

Comentarios:

Puntos fuertes del aprendiz:

Propuestas de mejora:

Valoración del proceso

Nada

Satisfactorio

Muy

a lo largo del curso

Satisfactorio

Satisfactorio

Satisfactorio

Calificación global

1

$2 \mid 3$

\begin{tabular}{l|l}
4 & 5
\end{tabular}

6

8

Firmas:

\begin{tabular}{|c|c|c|}
\hline Tutor/a centro escolar & Coordinador & Tutor/a Facultad \\
& Departamento de & Educación / \\
& Administración & \\
\hline
\end{tabular}


Estudios Pedagógicos, vol. XL, Número Especial 1: 29-48, 2014

LA EVALUACIÓN DE COMPETENCIAS EN UNA EXPERIENCIA DE FORMACIÓN DUAL DE MAESTROS:

DIFERENCIAS Y SEMEJANZAS ENTRE TUTORES DE ESCUELA Y DE UNIVERSIDAD

Anexo 4: Observación de intervención guiada de los tutores de la facultad / universidad

Actividad (título y/o breve descripción):

Grado en que se presenta el indicador

1. Gestión y organización de la actividad y del aula (clima) Nada $\quad$ Poco Bastante Mucho

1.1 Presenta la actividad de forma clara y el que se pretende con ella $\quad \begin{array}{llll}1 & 2 & 3\end{array}$

1.2 Organiza el tiempo para desarrollar la actividad prevista ......
1 $\quad 2 \quad 3 \quad 3 \quad 4$

1.3 Dinamiza el aula favoreciendo un clima de trabajo y de respeto $\quad \begin{array}{llll}1 & 2 & 3\end{array}$

1.4 Se muestra seguro/a y tranquilo/a ................................ $1 \quad 1 \quad 2 \quad 3 \quad 4$

\section{Desarrollo de la intervención. Actividades de E/A}

\subsection{Lenguaje}

2.1.1 Se expresa con un registro docente adecuado a la edad del alumnado

2.1.2 Utiliza el léxico adecuado en la actividad y los contenidos

2.1.3 Apoya la comunicación verbal con la no verbal

$\begin{array}{llll}1 & 2 & 3 & 4 \\ 1 & 2 & 3 & 4 \\ 1 & 2 & 3 & 4\end{array}$

\subsection{Interacción}

2.2.1 Correcta interacción y contacto con los/las alumnos ....

2.2.2 Refuerza y motiva positivamente a los alumnos ...

$\begin{array}{llll}1 & 2 & 3 & 4 \\ 1 & 2 & 3 & 4\end{array}$

\subsection{Actividades E/A}

2.3.1 La actividad es adecuada a los conocimientos previos .. $\quad \begin{array}{lllll}1 & 2 & 3 & 4\end{array}$

2.3.2 El ritmo de la actividad es adecuado

2.3.3 La dinámica promueve la actividad del alumnado

$\begin{array}{llll}1 & 2 & 3 & 4\end{array}$

2.3.4 Hace intervenciones oportunas para promover la progresión en la actividad .....

$\begin{array}{llll}1 & 2 & 3 & 4\end{array}$

$\begin{array}{llll}1 & 2 & 3 & 4 \\ 1 & 2 & 3 & 4\end{array}$

\subsection{Estrategias}

2.4.1 Utiliza ejemplos para potenciar la comprensión y el aprendizaje

2.4.2 Promueve la participación

2.4.3 Se muestra creativo/va en el desarrollo de la actividad favoreciendo el aprendizaje

\section{Evaluación}

3.1 Potencia en el alumnado la revisión para la corrección de su actividad

3.2 El alumnado sabe qué realiza correctamente y los aspectos que tiene que mejorar

\section{Recursos utilizados.}

4.1 Utiliza objetos, materiales, situaciones reales para reforzar su exposición

Comentarios:

Puntos fuertes del aprendiz en la intervención guiada:

Propuestas de mejora para el aprendiz

Firma del docente que hace la observación:

Firma del maestro del centro escolar 